\title{
TEST RESULTS OF AN INNOVATIVE AND MODULAR SENSOR DESIGN FOR FISSION CHAMBER STUDIES
}

\author{
G. de Izarra *, B. Geslot ${ }^{\dagger}$, S. Bréaud *, A. Pepino *, J-F. Villard *, P. Filliatre* \\ ${ }^{*}$ CEA, DEN, DER, Instrumentation, Sensors and Dosimetry Laboratory, \\ Cadarache, F-13108 St-Paul-Lez-Durance, France \\ ${ }^{\dagger}$ CEA, DEN, DER, LP2E, \\ Cadarache, F-13108 St-Paul-Lez-Durance, France
}

\begin{abstract}
Since many years, the Instrumentation, Sensors and Dosimetry laboratory at CEA Cadarache has been working to propose innovative and robust design of fission chambers. In this framework, few simulation tools like Chester or PyFc were developed to make detector prototyping and optimisation possible. Up to now, the code experimental validations are scarce mainly because of the lack of flexibility of regular fission chambers. In order to set up an experimental database for code validation, an innovative modular twin fission chamber, the CFTM, was designed. It allows to set with precision the inter-electrode gap, the filling gas pressure as well as the fissile deposit used. This detector was irradiated in Minerve zero power reactor in order to gather results for code validation. Thanks to a calibration procedure, a bank of experimental results is available for simulation validation.
\end{abstract}

\section{INTRODUCTION}

Since 2000's, the Instrumentation, Sensor and Dosimetry laboratory is involved in building simulation tools to make nuclear detectors prototyping and optimisation. Few of these tools were dedicated to fission chambers: Chester [1] were able to simulate current pulses at the output of the detector by using Monte-Carlo method. Additional simulation allowed to predict signal in current or in Campbell mode [2]. PyFc [3], another Monte Carlo based simulation was used to investigate fission chamber failure. Yet, most of the simulation tools lacked of experimental qualification. Few reasons may explain this. First, available fission chambers lack of modularity. It is not possible to reconfigure the detector since every part is welded. Second, there is uncertainties on some parameters like the filling pressure of the detector. Third, most of the measurements performed are not absolute since electronic systems are not calibrated in charge or in current.

An experimental work was made in order to produce data valuable for code validation. The major part of this effort consisted in the design of a new fission chamber which can be reconfigured on the fly. Additional work consisted in calibrating properly the measurement system to gather absolute charge and current estimations.

In the first part of the paper, the problem of code validation is discussed and the CFTM, a modular fission chamber is presented. Then, experiments on the Minerve zero power reactor are summarised. In a third part, simplified simulations are performed to link measured quantities to the one derived by simulation. At last, experimental results are discussed.

\section{EXPERIMENT DESIGN FOR CODE VALIDATION}

\section{A. Code validation}

Validation of a simulation tool is not straightforward especially if the range of validity of each model involved in the simulation and its uncertainty is needed. A global picture of the validation process is given in Fig. 1, simulation are made of a set of models and input data describing phenomena like transport of fission fragment in material, generation or drift of charged particles. Those are used to compute intermediate quantities like the amount of charges generated along each fission tracks or charge collection time which are usually not measurable directly. At last, those quantities make possible the estimation of the theoretical current pulses and the charges spectrum related to the detector of interest. Only the current pulses and the charges spectrum can be measured at the output of a fission chamber. N evertheless, by choosing c arefully the detector parameters which are reduced to its dimensions, the filling $g$ as $m$ ixture a nd the polarisation $v$ oltage, $i \mathrm{t} m$ ight be possible to retrieve intermediate quantities directly comparable to the simulation results.

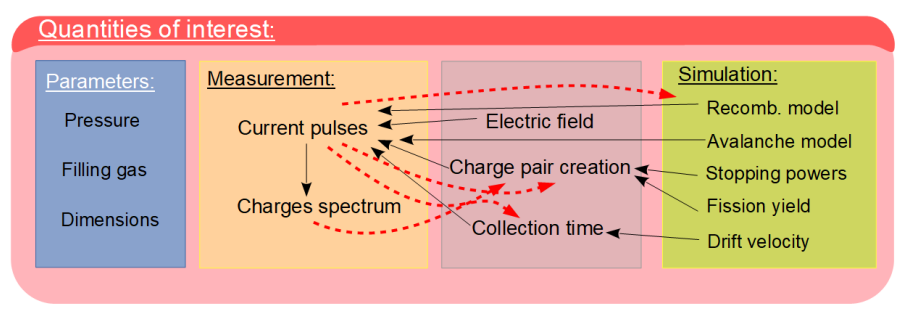

Fig. 1. Schema of the CFTM.

From that procedure, few design constraints might be derived to propose a fission chamber dedicated to experimental code validation:

- Reconfigurable detector: master the dimensions of the detector and their effects on signal. 
- Simple electric field: ability to keep the electric field simple regardless of the electrode configuration. Only one working regime during the drift of charged particles.

- Filling pressure between 1 and 5 bar: effect of the filling pressure on the signal.

- Limited effect of impurities on gas transport properties: master the electric charges drift and limit its uncertainty.

- Possibility to lose all the fission fragment energy in the gas: data on heavy ions kinetic energy losses and heavy ion distribution.

- Possibility to detect the two fission fragments emitted: information about fission yield and heavy ions energy losses.

- Possibility to calibrate with a reference reaction: absolute charge and current measurements.

- Possibility to work in saturation or recombination regime: information about recombination/stauration models.

\section{B. CFTM}

The design goals were used by the fission chamber workshop of CEA Cadarache to build a detector dedicated to code validation: the CFTM. This new detector consists of two flat fission chambers set back to back as shown in Fig. 2. It is constituted of a gastight titane body with two electric connectors, a tube for gas filling and a pressure s ensor port. The body is capped with a screwed lid to allow on the fly reconfiguration. Gas tightness is obtained by the use of nitrile $\mathrm{O}$ rings. The maximum filling pressure before lid deformation is 6 bars. The detector is modular: ionisation chambers are

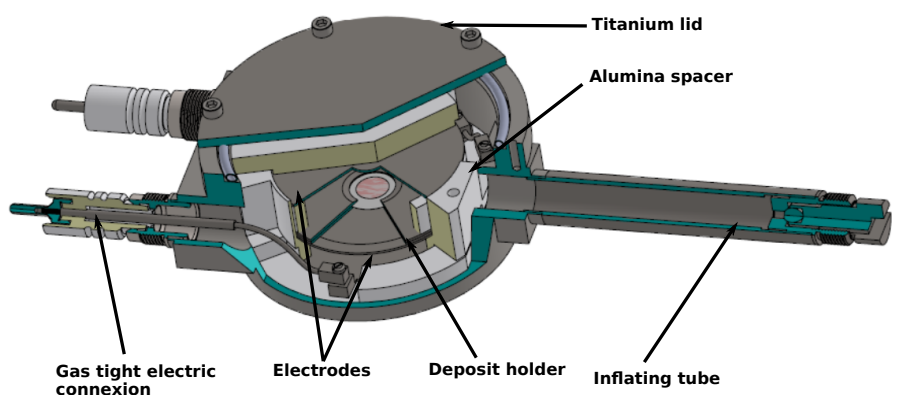

Fig. 2. Schema of the CFTM.

built by setting three electrodes (one central deposit holder and two regular electrodes) and alumina spacers in the body. The inner diameter of the ionisation chamber is $39 \mathrm{~mm}$ to allow a complete loss of energy of fission fragments within the 15 bar pressure range. The range of average light and heavy fragment in argon at a pressure of 1 bar in normal condition is respectively $R_{l}=25 \mathrm{~mm}$ and $R_{h}=18 \mathrm{~mm}$. From this data, it is clear that heavy ions may loose all their kinetic energy at filling pressure above 2 bars.

Thanks to sets of alumina spacers, electrodes can be arranged in three configurations: one with the deposit located in the centre of the detector with two $5 \mathrm{~mm}$ inter-electrodes gaps, one with a $9 \mathrm{~mm} / 1 \mathrm{~mm}$ inter-electrodes gaps, and a last one with $7.5 \mathrm{~mm} / 2.5 \mathrm{~mm}$ gaps. The two outer electrodes can be polarised to a different voltage through the two electric connexions while the fissile deposit holder and the detector body are grounded. The electric field is constant in each interelectrodes gap, it is given by:

$$
\overrightarrow{E_{\text {plan }}}=\frac{\Delta V}{L} \overrightarrow{e_{v}}
$$

where $\Delta V$ is the polarisation voltage and $L$ is the interelectrode distance.

Two different deposit holders were built, one is a $0.4 \mathrm{~mm}$ thick steel disc for back to back configuration while the other is constituted of two disks with a $9 \mathrm{~mm}$ diameter hole in their centre in order to sandwich thin foils of mylar or aluminium with fissile deposit on it.

The back to back configuration has many advantages, spectra ratio can be performed with a well known geometry and similar filling pressure. Alpha emitter deposits like ${ }^{238} \mathrm{Pu}$ or ${ }^{241} \mathrm{Am}$ might be used on one face of the detector to perform an absolute calibration of the charge pair generated by fission fragments in gas.
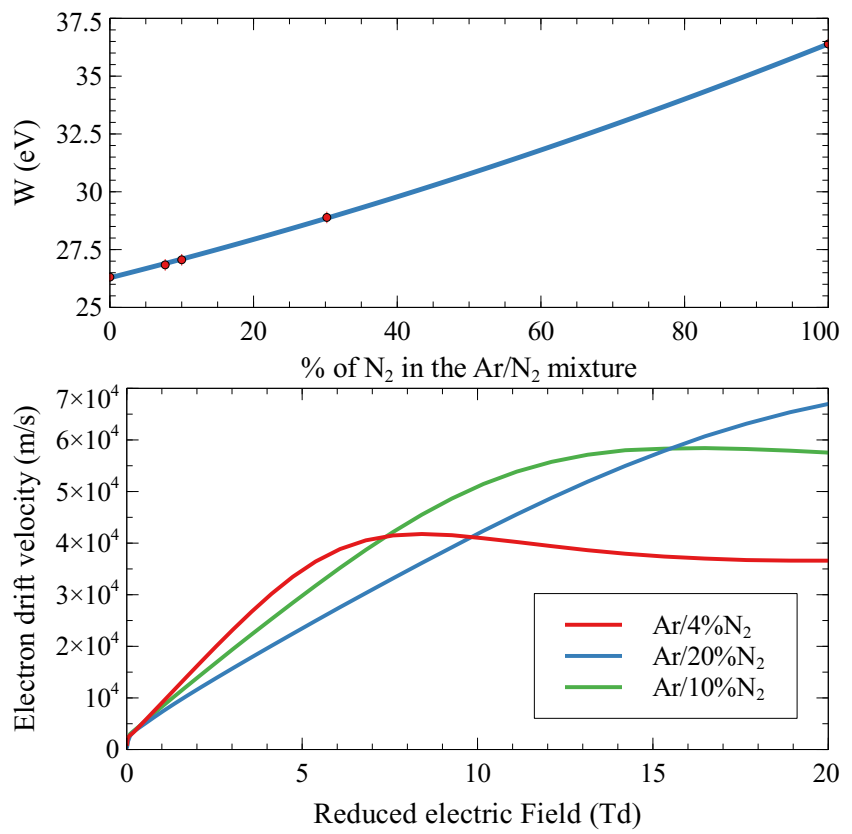

Fig. 3. Up: Mean energy needed to generate an electron/ion pair in an $\mathrm{Ar} / \mathrm{N}_{2}$ mixture as a function of the nitrogen proportion. Down: Electron drift velocty in various $\mathrm{Ar} / N_{2}$ mixtures. The reference filling gas for fission chamber is $\mathrm{Ar}+\% 4 \mathrm{~N}_{2}$.

Because of on the field reconfiguration, it is needed to fill the detector with a gas mixture almost insensitive to impurities from air. A study was made with different Argon/nitrogen mixtures to keep a high and constant electron drift velocity even with impurities. It was shown that $\mathrm{Ar}+20 \% \mathrm{~N}_{2}$ mixture is the best compromise between electron drift velocity and stability (fig.3). To monitor the filling gas pressure, a Keller M5 transducer is connected to the fission chamber body. It gives a pressure measurement with an accuracy of 0.01 bar. 


\section{Simulation}

The simulation of the detector is mandatory to determine the quantity of interest and how information can be gathered from experiments. A simplified simulation of the detector was built using the COSICAF simulation suite [4]. Because of the specific mixture used in the CFTM, it was necessary to expand the database of COSICAF. W values for argon nitrogen mixture were taken from Tawara \& al [5] while the electron drift velocity was computed by using BOLSIG software and the cross section from Phelps \& pitchford for nitrogen and Yamabe \& Buckman for argon [6]. The Moderation laws used for slowing down fission chamber in the gas were also computed thanks to PRAL algorithm and SRIM stopping powers [7].

Two pressures were investigated as well as two polarisation voltage: Direct polarisation, where the cathode is the deposit holder and inverse polarisation, where the anode correspond to the holder. There are few limitations in this simulation. First only two mean fission fragment are simulated, hence the pulse height spectrum fails to capture the effect of U235 fission yield. Second, COSICAF is only able to handle one heavy ion per fission which limits the simulation to the detector with thick fissile deposit. At last, the code is designed only to simulate current pulse at the output of detector working in saturation regime; It fails to take into account recombination regime.

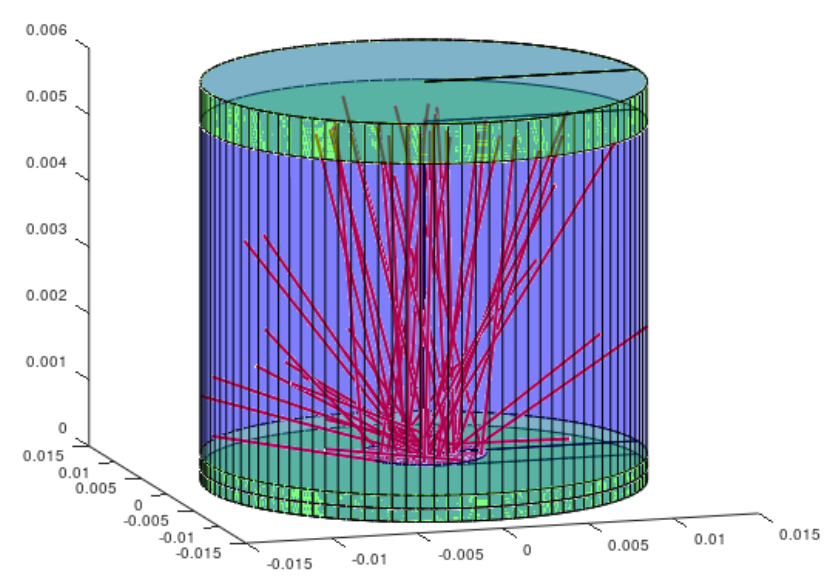

Fig. 4. Few fission f ragment $t$ rajectories c omputed in the CFTM geometry with COSICAF.

A picture of the simulated geometry is available in Fig. 4 with few fission fragments tracks.

Few pulses simulated with a direct polarisation are available in Fig. 5. Their amplitude is around $1 \mu \mathrm{A}$, which makes them distinguishable by using the typical CEA large bandwidth current preamplifier. I onic $p$ ulses a re $r$ eally $l$ ong and should not be distinguishable with current amplifier because of amplitudes of the order of nano amps. Nevertheless, electronic charges might be measured easily. Thanks to inverse polarisation, it is possible to estimate the charge carried by the ions during direct polarisation by gathering electrons back

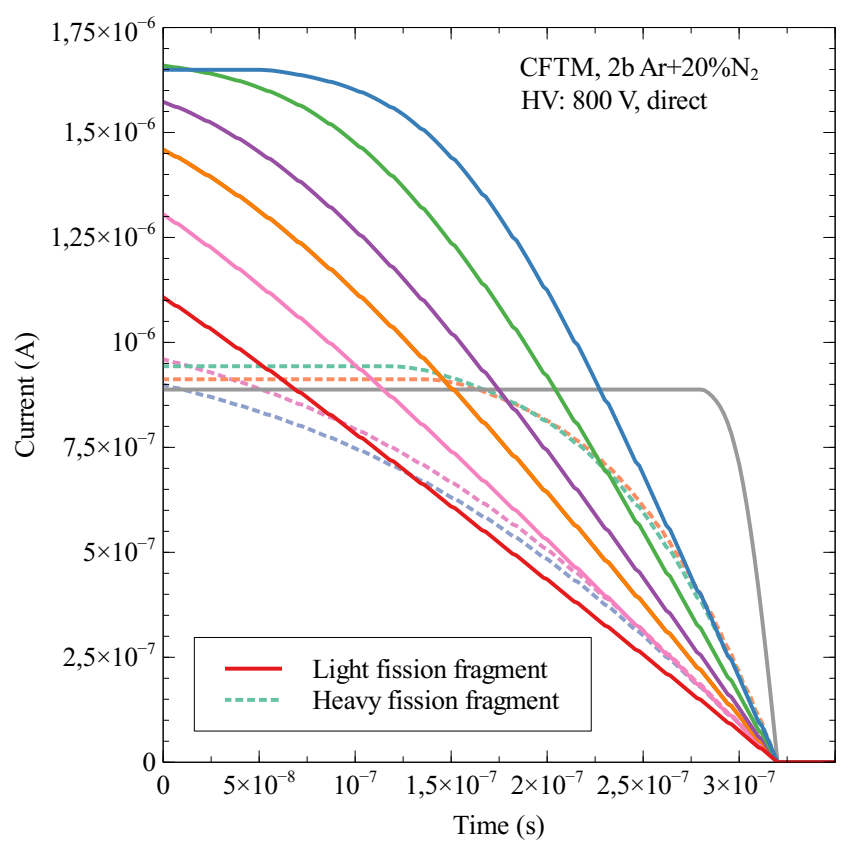

Fig. 5. Rapid simulation of CFTM current pulses in direct polarisation.

on the deposit holder. Useful quantities can be derived from pulses. First, the electron drift time is easily measurable since charge pairs are always generated at the vicinity of the cathode, thus, the pulse duration is linked to the time needed for those electrons to drift up to the anode. Multiple pulse shapes are distinguishable, triangular ones are related to heavy ion trajectories which hit the anode and rectangular pulses are characteristic of trajectories parallel to the electrode. Mixed rectangular/triangular shapes also exist, they are related to trajectories which are oriented toward the alumina wall.

From the plateau of rectangular pulses, it is possible to estimate the amount of charge pair generated along the fission fragment trajectory. It is given by:

$$
Q_{e}=\frac{i_{p}}{\overrightarrow{v_{d}}(E, N) \cdot \overrightarrow{E_{0}}},
$$

where $i_{p}$ is the plateau value, $Q_{e}$ is the total amount of electron generated along the fission fragment trajectory, $v_{d}$ is the electron drift velocity which depend of the electric field and particle density and $E_{0}$ the Shockley Ramo weighting field [8] wich in our case is constant and has similar orientation to the electric field.

Two examples of charge spectrum are depicted in Fig. 6 for a direct and an inverse polarisation. For direct polarisation, only one group of pulses is noticeable because most of the fission $\mathrm{f}$ ragments a re loosing o nly a $\mathrm{f}$ raction of $\mathrm{t}$ heir kinetic energy. In addition, they are producing the same order of magnitude of electrons at the vicinity of the fissile deposit. Since most of the induced charge is due to electrons close to the cathode, measurements give only a limited amount of information about fission y ield and difference of stopping powers. 
For inverse polarisation, two groups of pulses are clearly noticeable. They correspond to the two groups of fission fragments. This time, electrons generated far away from the fissile deposit are mostly responsible for the recorded charge. Since stopping powers of the fission fragments become more and more different while ions are loosing energy, inverse polarisation tend to exhibit the difference between heavy ions. Hence, inverse polarisation is the optimum configuration to get information on fission yield. and difference on stopping power.
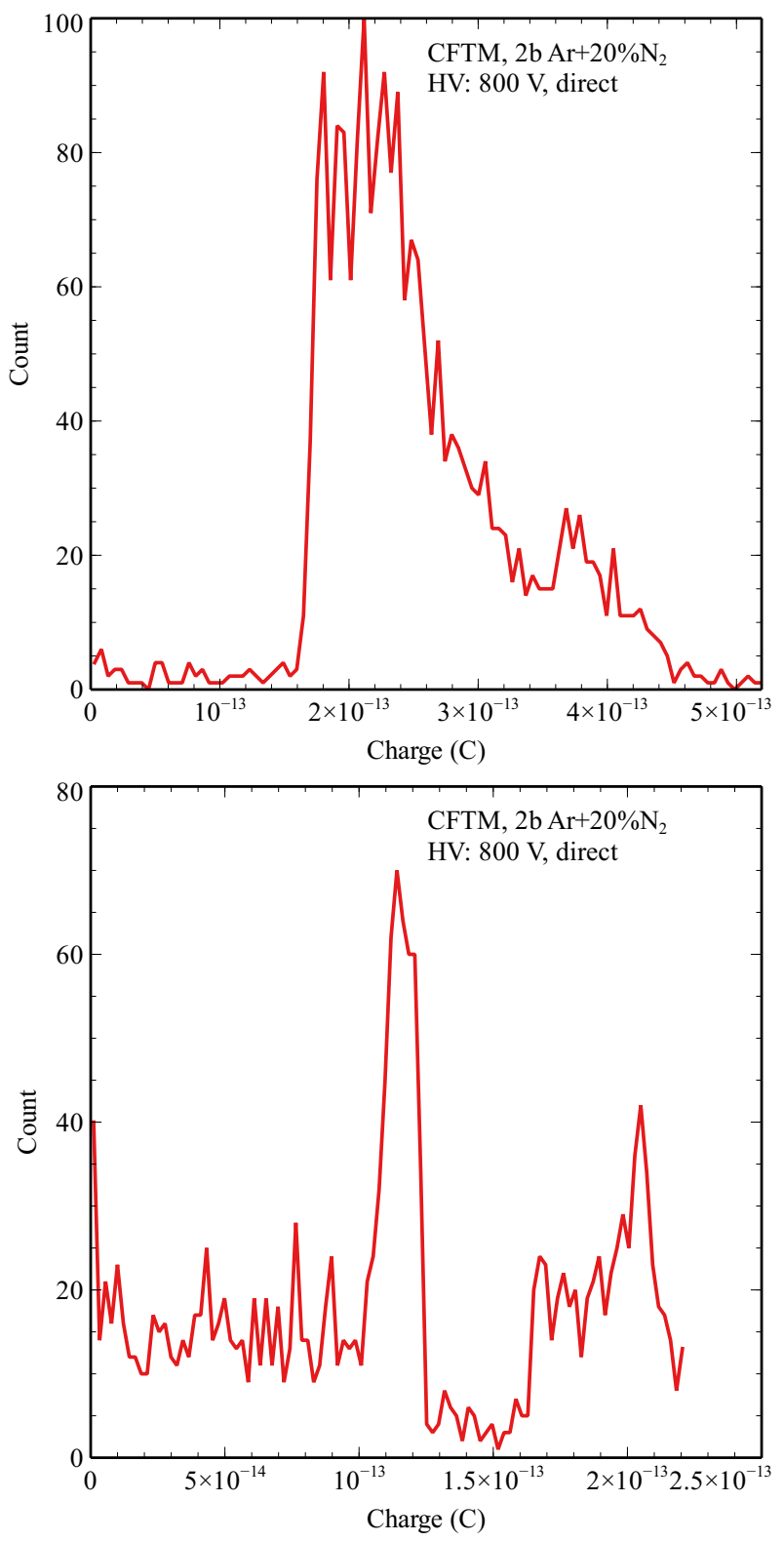

Fig. 6. Up: charge spectrum estimated with a direct polarisation. Down: charge spectrum simulated with an inverse polarisation.

\section{EXPERIMENTS}

An experimental campaign took place in the Minerve zero power reactor with two CFTM manufactured by the fission chamber workshop of CEA cadarache.

Minerve was a french pool type reactor located in CEA Cadarache. The core was constituted of two parts: a driver zone that contains aluminium/uranium plates assembly surrounded by graphite reflector and a central square cavity in the middle of the driver zone which received experimental lattices used to produce a specific neutron spectrum. It was submerged under 3 meter of water. The reactor could be operated to a maximum power of $100 \mathrm{~W}$, which correspond roughly to a neutron flux in the core center of around $10^{9}$ $\mathrm{cm}^{-2} \cdot \mathrm{s}^{-1}$. Irradiations were performed in a dry tube installed in the reactor reflector for the experiment. Because of its large dimensions, the detector was installed in a dummmy reflector assembly.

Two instrumentations sensitive to charge and to current were used during the experiment. One has two channels with a Canberra PA2006 and a Ortec 142 preamplifiers connected respectively to Canberra amplifier A2024 and A2026. Both channels were connected to a canberra MP2 multichannel analyser. The other electronic instrumentation consisted of two current preamplifiers designed at CEA with a bandwith of $\approx 100 \mathrm{MHz}$. To keep a reasonable energy resolution, 6 meter of RG58 cable with an estimated capacity of $680 \mathrm{pF}$ were used to connect preamplifiers to the CFTM.

\section{A. Fission chamber configurations}

Two different detectors were assembled. Each had a symmetric electrode configuration with $5 \mathrm{~mm} / 5 \mathrm{~mm}$ inter-electrode gaps. The first detector, P2 (Fig.7), contains a thick deposit holder with a $0.78 \mathrm{~cm}^{2} \mathrm{U}_{3} \mathrm{O}_{8}$ deposit. The deposit contains $10 \mu \mathrm{g}$ of $\mathrm{U} 5$ to reach a count rate of around $1000 \mathrm{cps}$ at reactor full power.

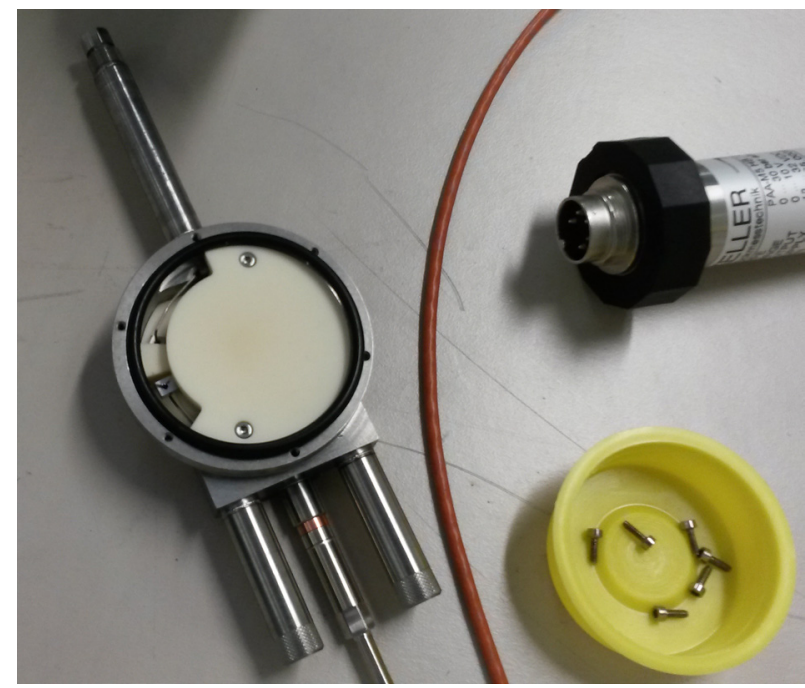

Fig. 7. Picture of a CFTM being configured.

The second detector, P3, was built to detect both fragments produced during a fission. This time the deposit consists of an evaporated droplet of uranyl nitrate sandwiched between two $2 \mu \mathrm{m}$ thick PET foils. 
For preliminary tests, P2 and P3 were filled $\mathrm{w}$ ith a $\mathrm{Ar}+4 \% \mathrm{~N}_{2}$ mixture usually used in regular fission chamber. It was shown that electron drift velocity is less sensitive to gas impurity with a high content of nitrogen in the mixture. Adding more quenching gas also rise the reduced field needed for electron avalanche. As a matter of fact, most of the experiments were conducted with a $\mathrm{Ar}+20 \% \mathrm{~N}_{2}$ mixture as the filling gas.

Numerous pressure and polarisation voltage were investigated to exhibit the recombination and the saturation regime as well as detection of both electronic and ionic charges.

\section{REsults}

Only a few experimental results obtained with current preamplifier a nd $\mathrm{w}$ ith $\mathrm{P} 2 \mathrm{a}$ re $\mathrm{p}$ resented $\mathrm{i} \mathrm{n} \mathrm{t}$ he following section. To have absolute measurements, each preamplifier was calibrated. First, a simulation using SPICE software was made to estimate the theoretical transfer function. Even if the transimpedance order of magnitude is good, real preamplifier's gain seem to departure from the computed value because a stage of the preamplification is tunable through potentiometer. We then decided to use a arbitrary wave generator and a well characterized current injection device to measure the current preamplifier transimpedance. Pulses recorded with P2 detector polarised at $700 \mathrm{~V}$ with a filling pressure of 2 bar are shown in Fig. 8. Rectangular and triangular pulse shapes are noticeable as predicted by the simulation. Signal to noise ratio, between 2 and 6 , is sufficient to compute the induced charge with precision, but make the estimation of charged particles drift time and rectangular pulses plateau values difficult.

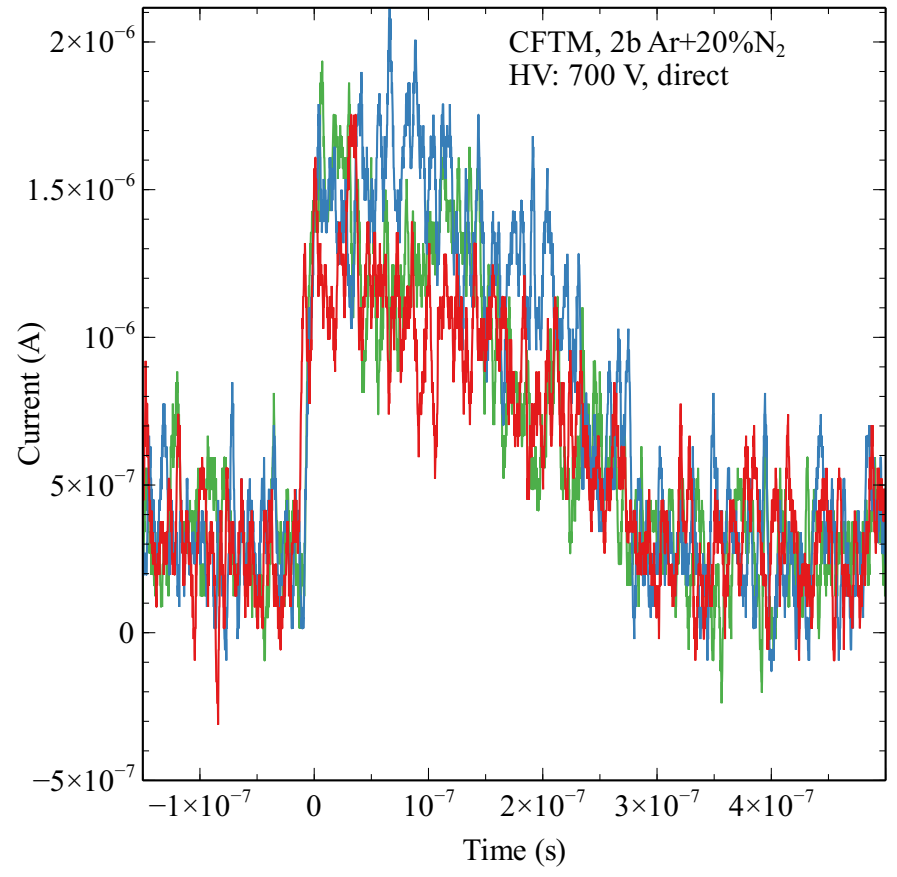

Fig. 8. Example of CFTM current pulses.
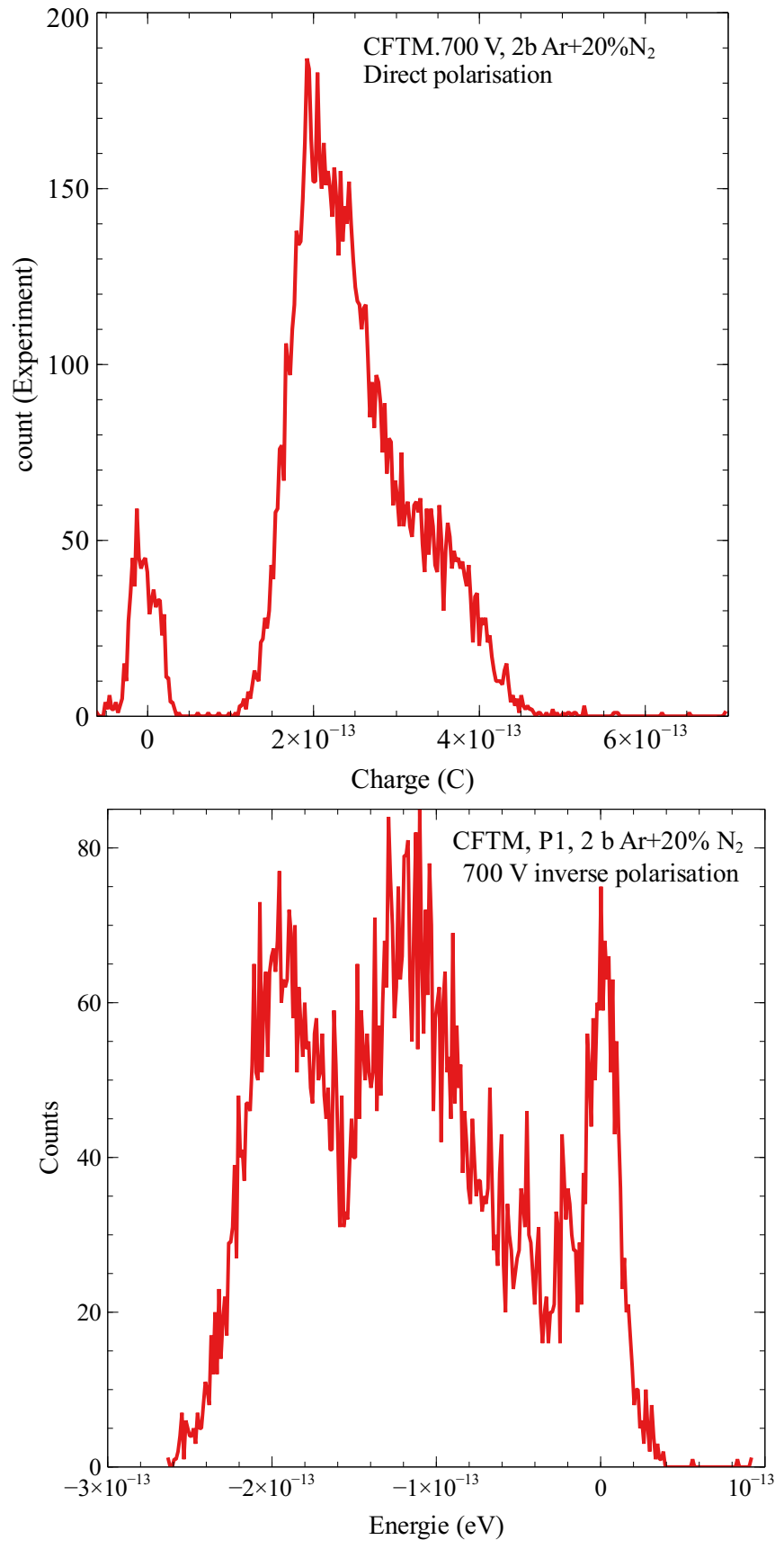

Fig. 9. Up: charge spectrum estimated with a direct polarisation. Down: charge spectrum simulated with an inverse polarisation.

Computed charge spectrum are shown in Fig. 9. The charge has an identical shape as the one from Fig. 6. Moreover, the mean charge value is estimated to $2.53 \pm 0.1010^{-13} \mathrm{C}$ while the mean charge estimated by the simulation with the same condition is $2.5110^{-13}$. Other derived quantities like the drift time and the plateau value are available in Table $\mathrm{I}$. Uncertainties on those values don't allow to validate the simulation code since uncertainty on drift velocity and fission fragments energy losses models involved in software are usually lower. 
TABLE I

DRIFT TIME AND PLATEAU VALUE OF THE EXPERIMENT CONDUCTED WITH P2 PROTOTYPE FILLED WITH AR+ $20 \% \mathrm{~N}_{2}$.

\begin{tabular}{|c|c|c|c|}
\hline Detector & E/N (Td) & exp. drift time (s) & plateau value (A) \\
\hline P2 2b, 700 V & 2.83 & $(3.14 \pm 0.32) 10^{-7}$ & $1.0910^{-06}$ \\
\hline P2 1b, 400 V & 3.23 & $(3.09 \pm 0.43) 10^{-7}$ & $8.0310^{-07}$ \\
\hline P2 4b, 1000 V & 2.02 & $(4.12 \pm 0.45) 10^{-7}$ & - \\
\hline
\end{tabular}

A global validation of the simulation is still possible thanks to the charge spectra. Since it is an integrated quantity, it is immune to high frequency noise. Moreover, the transimpedance of the preamplifier is known with an uncertainty of $4 \%$. Deviation of spectra mean values and other moments give hints on model discrepancies.

\section{CONCLUSION}

A new detector, the CFTM, was designed and built for simulation validation purpose. Thanks to its reconfigurability, we showed it is possible to access intermediate quantities generated by simulation like charge pairs created or drift velocity. An experimental campaign took place in Minerve zero power reactor with the CFTM. At this occasion numerous data were gathered at different polarisation voltage and filling gas pressure. The calibration of the electronics measurement system allowed to estimate the absolute charge and current at the output of the detector. Unfortunately, due to our measurement system limitations, the signal to noise ratio is too low to provides most of the quantities awaited with a precision good enough to validate simulation codes. Still, it is possible to assess the global accuracy of a simulation with the help of charge spectra. To enhance the results, new measurements have to be done with a higher polarisation Voltage. An increase of the signal to noise ratio of a factor 2 is awaited while keeping the detector in a saturation regime. An enhanced smoothing procedure based on simulated results and spline approximation is also tried in order to improve the drift time and the plateau value evaluation.

\section{REFERENCES}

[1] P. Filliatre, C. Jammes, B. Geslot, and R. Veenhof, "A monte carlo simulation of the fission chambers neutron-induced pulse shape using the garfield suite," Nuclear Instruments and Methods in Physics Research Section A, vol. 678, pp. 139-147, 2012.

[2] P. Filliatre and C. Jammes, "A comprehensive numerical modelling of a fission chamber to be operated over a wide dynamics in the vessel of a sodium-cooled reactor," in Physor 2016 proceedings, 2016.

[3] Z. Elter, "pyfc: a trim-based fission chamber pulse shape simulator," CHALMERS UNIVERSITY OF TECHNOLOGY, Tech. Rep., 2015.

[4] G. de Izarra, "Cosicaf software," https://github.com/gdeIzarra/cosicaf, 2019.

[5] H. Tawara, J. Kikuchi, and T. Doke, "Energy dependences of W values for $\mathrm{MeV}$ alpha particles in $\mathrm{Ar}-\mathrm{Xe}, \mathrm{Ar}-\mathrm{N}_{2}$ and $\mathrm{Ar}-\mathrm{CH}_{4}$ mixtures," $\mathrm{NIMB}$, 1986.

[6] G. Hagelaar and L. Pitchford, "Solving the boltzmann equation to obtain electron transport coefficients and rate coefficients for fluid models," Plasma Sources Sci. Technol, vol. 14, pp. 722-733, 2005.

[7] J. Ziegler, M. Ziegler, and J. Biersack, "Srimthe stopping and range of ions in matter (2010). nuclear instruments and methods," Nuclear Instruments and Methods in Physics Research Section B: Beam Interactions with Materials and Atoms, vol. 268, pp. 1818-1823, 2010.
[8] Z. He, "Review of the ShockleyRamo theorem and its application in semiconductor gamma-ray detectors," Nuclear Instruments and Methods in Physics Research A, vol. 463, pp. 250-267, 2001. 